\title{
Livia Prüll, Christian George, Frank Hüther (Hg.), Universitätsgeschichte schreiben
}

\section{Antonin Dubois}

\section{OpenEdition}

\section{Journals}

Édition électronique

URL : http://journals.openedition.org/ifha/10672

DOI : $10.4000 /$ ifha. 10672

ISSN : 2198-8943

\section{Éditeur}

IFRA - Institut franco-allemand (sciences historiques et sociales)

\section{Référence électronique}

Antonin Dubois, «Livia Prüll, Christian George, Frank Hüther (Hg.), Universitätsgeschichte schreiben » Revue de l'IFHA [En ligne], Date de recension, mis en ligne le 28 avril 2020, consulté le 24 septembre 2020. URL : http://journals.openedition.org/ifha/10672 ; DOI : https://doi.org/10.4000/ifha.10672

Ce document a été généré automatiquement le 24 septembre 2020

(CIFHA 


\title{
Livia Prüll, Christian George, Frank Hüther (Hg.), Universitätsgeschichte schreiben
}

\author{
Antonin Dubois
}

\section{RÉFÉRENCE}

Livia Prüll, Christian George, Frank Hüther (Hg.), Universitätsgeschichte schreiben. Inhalte - Methoden - Fallbeispiele, Göttingen/Mainz : V\&R unipress/Mainz University Press, 2019, 255 p., $40 €$ 
L'histoire des universités est, sinon un courant, du moins un domaine de recherche bien établi en Allemagne. En témoigne l'existence d'une Gesellschaft für Wissenschafts- und Universitätsgeschichte ou d'un Forschungsverbund für Universitätsgeschichte à l'université de Mayence. Les membres de ce dernier ont organisé le colloque à l'origine du présent ouvrage. Le Forschungsverbund entend plus précisément favoriser les recherches sur l'université de Mayence. Cet intérêt pour l'histoire individuelle d'une université est ancien. Depuis le $\mathrm{XIX}^{\mathrm{e}}$ siècle, des Festschriften (ouvrages commémoratifs) historiques sont publiés lors du jubilé d'une université, tradition encore rare en France.

De la fondation des premières universités au Moyen Âge à leur transformation en universités de masse à la fin du $\mathrm{XX}^{\mathrm{e}}$ siècle, en passant par la constitution des Landesuniversitäten par les souverains de nombreux États composant le Saint-Empire romain germanique à l'époque moderne, ou encore la place des universités durant le Troisième Reich : toutes les périodes ont été abordées, à travers divers prismes, comme l'histoire institutionnelle et des structures, des professeurs, des étudiants, des disciplines. Il n'est donc pas surprenant que les historiennes et historiens allemands considèrent le temps venu de faire le bilan de ces recherches et des méthodes employées pour les mener. Quelques articles dressant un état de l'art (Sylvia Paletschek en 2011 dans NTM Zeitschrift für Geschichte der Wissenschaften, Technik und Medizin) ou de réflexion sur l'écriture de l'histoire des universités (Stefan Gerber et Marian Füssel en 2014 dans la même revue) ont précédé cet ouvrage collectif, qui porte principalement sur l'Allemagne mais inclut également l'Autriche et la Suisse germanophone.

Après une introduction de Livia Prüll, qui expose le projet du Forschungsverbund de Mayence et présente l'ouvrage, Rainer Christoph Schwinges dresse un vaste panorama de l'histoire de l'Universitätsgeschichte. L'historien suisse, qui a lui-même publié ou dirigé de très nombreux ouvrages dans ce domaine, prend pour point de départ le vaste projet d'une Histoire de l'université en Europe, sous la direction de Walter Rüegg. Les quatre volumes de cette Histoire ont été publiés en allemand, anglais, espagnol, portugais, russe et chinois, mais pas en français, ni en italien. C. Schwinges revient notamment sur la périodisation choisie (qui suit quatre grands moments de réformes : premières universités au Moyen Âge, humanisme au XVI ${ }^{\mathrm{e}}$ siècle, projet de Wilhelm von Humboldt au début du XIX ${ }^{\mathrm{e}}$ siècle et démocratisation des universités à partir de 1945) et sur la structure commune aux quatre volumes (panorama général, structures, étudiants, disciplines). Il dresse ensuite non seulement un panorama des publications, en insistant sur les plus récentes, mais également des lieux de ces publications, comme les revues ou les collections, les sources et leur édition. 
Si des travaux ont été publiés auparavant, C. Schwinges considère que ce n'est qu'à partir de la fin du $\mathrm{XX}^{\mathrm{e}}$ siècle que ce domaine de recherche, dans le sens d'une histoire scientifique et critique, se développe réellement. Les transformations profondes et rapides de l'enseignement supérieur, la confrontation des historiennes et historiens avec le rôle de leurs prédécesseurs sous le nazisme, les mouvements étudiants des " années 68 » sont autant de facteurs explicatifs à cet intérêt renforcé pour l'histoire des universités. C. Schwinges distingue aujourd'hui quatre domaines principaux: les universités comme institutions et leurs constitutions (Verfassungen); les personnes qui les font fonctionner avec des biographies ou prosopographies de professeurs ou de diplômés par exemple, leur conscience (ou habitus) professionnelle et académique, leur intégration aux élites; les étudiants et en particulier leur mobilité ; l'image extérieure de l'université et sa communication publique. Ces différentes thématiques sont inégalement réparties entre les périodes. Le dernier thème cité est par exemple surtout travaillé pour l'époque moderne.

Après ce vaste panorama introductif, une première partie est consacrée aux transformations des universités du Moyen Âge à la fin $d u X X^{e}$ siècle et à leur historiographie. Le terme "Universitätsgeschichte» est ici compris dans le double sens d'histoire des institutions universitaires et de l'écriture de cette histoire. Les quatre chapitres dressent en réalité surtout un portrait synthétique du système universitaire allemand à la lumière de l'historiographie (sujet sur lequel nous ne pouvons nous étendre ici), et insistent assez peu sur les évolutions de celle-ci. Le découpage chronologique des quatre chapitres témoigne non seulement de problématiques spécifiquement allemandes, mais d'un choix éditorial de mettre en avant la question de la « démocratisation » de et à l'université après la Seconde Guerre mondiale. En effet, dans son introduction, Livia Prüll indique que le second $\mathrm{XX}^{\mathrm{e}}$ siècle représente un « défi particulier pour l'histoire des universités » en raison des nombreux «bouleversements politiques » (p. 15-16). De ce fait, le premier chapitre va du Moyen Âge à 1933, tandis que les trois autres portent sur une partie du $\mathrm{XX}^{\mathrm{e}}$ siècle: le Troisième Reich, l'Allemagne de l'Ouest entre 1945 et les années 1970-1980, la République démocratique allemande.

Martin Kintzinger considère comme une première forme d'écriture de l'histoire des universités les textes des universitaires du Moyen Âge et surtout de l'époque moderne qui reviennent sur leur expérience, parfois avec une perspective critique ou programmatique sur la science ou la politique. Toutefois, il voit dans le Räsonnement über die protestantischen Universitäten in Deutschland de l'orientaliste et théologien Johann David Michaelis, publié entre 1768 et 1776, le véritable commencement d'une histoire des universités dans l'espace germanophone (p. 59). Pendant plus d'un siècle cohabitent textes d'intervention et de réflexion (par exemple sur la fondation de l'université de Berlin ou les discours d'entrée en fonction des recteurs successifs de chacune des universités), souvenirs, chroniques et études historiques. Ces publications portent principalement sur les universités allemandes, mais aussi parfois étrangères, comme l'ouvrage de Victor Aimé Huber sur l'Angleterre (1839/40). Vers 1900, le nombre de publications historiques augmente considérablement.

Michael Grüttner souligne au début de sa contribution que le Troisième Reich constitue probablement la période la mieux étudiée en ce qui concerne l'histoire des universités. Il distingue quatre phases dans le développement de ces travaux : 1) de 1945 à la fin des années 1950 où le sujet est l'objet de débats médiatiques mais pas de recherches 
historiques; 2) les années 1960-1970 où le sujet oppose politiquement étudiants et professeurs dans le cadre de la remise en cause de l'Ordinarienuniversität ; 3) les années 1980 sont une phase de transition où paraissent tant des ouvrages commémoratifs taisant ou minimisant la période nazie que de premiers travaux empiriques et critiques ; 4) des années 1990 à nos jours, période d'inflation du nombre de publications et d'importants débats suite à la révélation du passé nazi d'universitaires de premier ordre, comme les historiens Theodor Schieder et Werner Conze.

Barbara Wolbring, autrice d'un ouvrage sur l'université dans les discours réformistes dans les zones d'occupation française, britannique et américaine entre 1945 et 1949, revient dans son article sur le rôle (auto-)attribué à l'université dans le processus de démocratisation de la société allemande après la Seconde Guerre mondiale. Ce n'est qu'à partir des années 2000 que des travaux ont souligné les entreprises de réforme du système universitaire dès le tournant des années 1950-1960, tandis que dominait jusqu'alors l'image de conservatisme et d'immobilisme jusqu'aux révoltes étudiantes de la fin des années 1960. Pour B. Wolbring, c'est l'immédiat après-guerre qu'il faut prendre en compte : selon elle, ne s'opposent pas alors que pro- et anti-réformistes, mais également des projets de réforme antagonistes, portés notamment par des universitaires d'un côté et par des représentants politiques de l'autre. Deux débats principaux, qui perdurent jusqu'à nos jours, s'imposent à ce moment: la fin de l'élitisme social et la démocratisation des structures universitaires. Toutefois, B. Wolbring doit concéder que malgré quelques tentatives, les réelles réformes ne débutent que progressivement dans les années 1960.

Ilko-Sascha Kowalczuk souligne en introduction que deux positions se font face sur la manière dont est traité le passé des établissements d'enseignement supérieur de l'exRDA : certains, comme Peer Pasternack, considèrent qu'il existe un grand nombre d'établissements ce qui explique leur traitement inégal, d'autres, comme I.S. Kowalczuk, jugent de manière générale ce traitement comme insuffisant, trop peu critique et ayant très rarement "su honorer ou même réhabiliter les persécutés " (p. 124). On peut se demander si là est réellement le rôle des historiens et historiennes. I.-S. Kowalczuk traduit ici son expérience personnelle : né en 1967 à Berlin-Est, il n'est pas autorisé à étudier, obtient son Abitur dans une école du soir en 1988 tout en travaillant parallèlement comme ouvrier, puis peut entreprendre des études d'histoire après la chute du Mur. Il ne revient pas plus avant sur ce débat, mais discute de manière critique les ouvrages publiés à l'occasion du sixième centenaire de l'université de Leipzig (2009) et du bicentenaire de l'université Humboldt de Berlin (2010), qu'il considère comme inédits dans leur traitement de la période communiste, mais médiocres dans leur démarche (ne mettant pas suffisamment en avant la mainmise du parti unique sur l'université notamment), et pauvres en ce qui concerne la période de transition des années suivant la chute du Mur. Notons que I.-S. Kowalczuk est lui-même auteur d'un des chapitres sur l'université Humboldt, qu'il semble renier au moins en partie, en citant longuement une recension très critique de Bernd Florath (note 65, p. 142-143). Il est d'ailleurs bien plus critique sur l'ouvrage berlinois que sur celui sur Leipzig, soulignant par exemple que la plupart des auteurs du tome 6 sur les disciplines ont « évité de se rendre aux archives » (p. 147).

La deuxième partie traite des méthodes et outils de l'histoire des universités. Christa Klein revient premièrement sur la biographie et la prosopographie. Elle use d'une définition particulièrement réduite de la méthode prosopographique (liste de noms avec quelques informations biographiques, dans une dimension strictement 
quantitative) pour mettre en avant la méthode de la «biographie collective » mêlant quantitatif et qualitatif. Elle amalgame pour cela des travaux très divers, dont certains datés, et dont plusieurs sont des études strictement statistiques et quantitatives qui ne se réclament aucunement de la prosopographie. Ce qu'elle dit de la biographie collective correspond en réalité à la démarche suivie par la plupart des travaux prosopographiques. Pour elle, une biographie collective porte sur 40 à 50 personnes au plus, tandis qu'une prosopographie traiterait d'ensembles plus importants - sans dire en quoi cette limite est justifiée, ni pourquoi le curseur est placé à 40 . On touche ici à une limite des frontières nationales des références, puisque La République des universitaires de Christophe Charle (1994) - pour ne citer qu'un ouvrage sur le thème de l'université - a fait de longue date la démonstration qu'une prosopographie peut mêler quantitatif et qualitatif. Christa Klein revient ensuite sur la démarche adoptée dans sa thèse de doctorat sur la Faculté de philosophie de Fribourg-en-Brisgau entre 1945 et 1970, dans laquelle elle a combiné données statistiques, analyse prosopographique (ou de "biographie collective») des professeurs et biographie plus poussée de certains d'entre eux, en s'interrogeant sur leur rapport à l'espace public.

Christian George, directeur des archives de l'université de Mayence, retrace l'histoire de l'institution qu'il dirige et souligne l'intérêt de ces archives universitaires pour la recherche historique. Ces Universitätsarchive - qui font défaut en France - ont presque le «monopole » de la conservation des fonds des universités. Elles disposent également parfois des archives d'associations ou de personnalités liées à l'université. L'auteur cite plusieurs exemples de projets menés grâce à la coopération entre chercheurs et archivistes, comme la prosopographie des mathématiciennes et mathématiciens allemands entre 1920 et 1960 à l'université de Wuppertal.

Livia Prüll, historienne de la médecine, revient sur le rapport entre histoire des universités et histoire des sciences, et plaide pour un rapprochement renforcé de ces deux courants. Bien qu'extrêmement général - renvoyant à des auteurs aussi différents que Karl Lamprecht, Karl Popper, Bruno Latour ou Thomas Kuhn -, curireusement, le début de son article n'évoque pas les travaux d'historiographie, nombreux en Allemagne, comme une combinaison réussie d'histoire des universités et des sciences. Pourtant, le premier des deux cas concrets censés montrer la fécondité de cette alliance porte sur l'historien Gerhard Ritter, et plus précisément sur sa conférence «L'idée de l'université et la vie publique». L. Prüll précise bien que la personne de G. Ritter permet de croiser histoire de l'université et de la discipline historique (p. 208). Dans cette conférence d'avril 1945, G. Ritter plaide pour le renouveau de l'idéal humboldtien de la liberté de la science et de l'enseignement. Il cherche ainsi à réhabiliter l'université allemande compromise sous le nazisme (fait considéré par L. Prüll comme relevant de l' Universitätsgeschichte) et illustre les prises de position publiques caractéristiques des historiens de son temps (que l'autrice voit comme relevant de la Wissenschaftsgeschichte). On remarque là à quel point les subdivisions en courants ou en domaines historiques peuvent être artificielles. Le second exemple, le professeur de médecine Ludwig Heilmeyer, ses succès en laboratoire et auprès des étudiants, ses nombreuses interventions médiatiques, n'est guère plus convaincant quant à la pertinence de la division entre les domaines relevant de l'histoire des universités ou celle des sciences.

Deux études de cas locales viennent compléter dans une troisième partie ces perspectives. Elles démontrent à nouveau, si besoin était, l'importance des Festschriften 
- certes d'un niveau scientifique réhaussé - dans l'écriture de l'histoire des universités germanophones. Mitchell G. Asch revient précisément sur l'organisation du $650^{\mathrm{e}}$ anniversaire de l'université de Vienne en 2015 et sur l'imposant ouvrage publié à cette occasion. Thomas Becker retrace quant à lui le difficilement établissement d'une histoire officielle de l'université de Bonn, fondée en 1818. Ni les ouvrages de son centenaire ni ceux de son cent cinquantenaire n'ont pu paraître en entier. La publication commémorant le bicentenaire est la première à voir le jour dans son intégralité. Elle est divisée en deux parties de plusieurs volumes chacune : une histoire générale de l'université et une histoire des facultés, ou plus exactement une histoire des sciences des différentes disciplines enseignées dans ces facultés.

L'ensemble des contributions s'appuie sur une riche littérature. Précisons que l'immense majorité de cette littérature est en langue allemande, ce qui s'explique largement par l'absence de comparaisons ou de perspectives internationales. L'absence d'une bibliographie générale en fin d'ouvrage - qui aurait mérité d'être une bibliographie ordonnée - est un véritable manque. Une telle bibliographie aurait été un apport important pour le livre, vers lequel il aurait pu être aisé de se reporter ou de renvoyer pour la recherche de références. J'ai relevé 154 références historiographiques dans la seule contribution de Christoph Rainer Schwinges (sans compter les renvois à différents articles d'un même numéro de revue ou ouvrage collectif, ni les renvois vers d'autres chapitres du présent livre). Devant une telle profusion de références, il est très difficile de retrouver un titre, en particulier dans des notes de bas de page atteignant ou dépassant régulièrement une demi-page.

Universitätsgeschichte schreiben est un ouvrage bien plus descriptif qu'analytique, aux contributions très voire trop diverses - ce qui explique qu'il ait fallu revenir sur chacune d'elles - et de qualité inégale. Il intéressera celles et ceux qui mènent des recherches sur les universités allemandes, particulièrement du (second) $\mathrm{XX}^{\mathrm{e}}$ siècle, ou qui comptent s'y engager. Écrit par et pour des chercheuses et chercheurs internes à ce champ de recherche, il manque (parfois cruellement) de références extérieures et souvent de regard critique. Seul un point de vue externe - ici un point de vue français informé sur l'historiographie de ce champ - permet de combler en partie ce manque, en attendant la publication d'un tel ouvrage proposant bilans et perspectives au niveau européen.

\section{INDEX}

Index chronologique : Époque contemporaine, Ouvrages transpériodiques

Mots-clés : Histoire des idées, Historiographie 
AUTEURS

ANTONIN DUBOIS

EHESS 